\title{
The latest CMS results on Higgs Boson decaying to two Photons with $13 \mathrm{TeV}$ data
}

\author{
Arnab Purohit* on behalf of the CMS Collaboration \\ Saha Institute of Nuclear Physics, HBNI, Kolkata, India \\ E-mail: arnab.purohitecern.ch
}

The analysis, reported here, to measure attributes of the Standard Model Higgs boson using the $\mathrm{H} \rightarrow \gamma \gamma$ decay channel, uses the data which corresponds to an integrated luminosity of 35.9 $\mathrm{fb}^{-1}$ collected by the CMS experiment in proton-proton collisions during the 2016 LHC running period. The measured combined signal strength relative to the standard model prediction is $1.16_{-0.14}^{+0.15}=1.16_{-0.10}^{+0.11}(\text { stat } .)_{-0.08}^{+0.09}(\text { syst } .)_{-0.05}^{+0.06}$ (theo. $)$. Signal strengths associated with the different Higgs boson production modes are also measured. Estimated results of the coupling strength modifiers, in the kappa framework, of the Higgs boson to vector bosons and fermions and the same to photons and gluons are reported.

The European Physical Society Conference on High Energy Physics

5-12 July, 2017

Venice

*Speaker. 


\section{Introduction}

In Run-I (2010-2013) of the Large Hadron Collider's (LHC) p-p collision, at center-of-mass energy $(\sqrt{s})$ of 7 and $8 \mathrm{TeV}$, the long sought Standard Model (SM) Higgs boson, through it's diphoton decay channel, was discovered. In July 2012, almost half a centuary from Higgs boson's theoretical developements, in the two large particle detectors, ATLAS and CMS, of LHC, a new particle whose properties are most consistent with SM Higgs boson, with mass around $125 \mathrm{GeV}$, was announced to be detected. The early Run-II data, recorded during 2015-2016, was analysed to measure the mass and other properties of the SM Higgs Boson more precisely than before. In this report the properties of the SM Higgs boson utilizing it's diphoton decay channel, analysing the data collected by the CMS detector, is presented. The analysis [1] is based on integrated luminosity of $35.9 \mathrm{fb}^{-1}$ of p-p collision data collected at $\sqrt{s}=13 \mathrm{TeV}$ during 2016. In the LHC environment the dominant production modes of the SM Higgs boson are gluon-gluon fusion ( $\mathrm{ggH})$, vector boson fusion (VBF), and associated production with a $\mathrm{W}$ or $\mathrm{Z}(\mathrm{VH})$ or $t \bar{t}$ pairs $(t \bar{t} H)$. The clean final state topology of the diphoton decay channel and high energy resolution of photons in the CMS detetor allows the mass of the Higgs boson to be reconstructed with high precision. However there is large background originating from irreducible background processes producing two prompt $\gamma$ and reducible background processes producing one prompt photon and a neutral meson, where the meson is misidentified as a $\gamma$ and two misidentified neutral mesons, which are reconstructed as $\gamma$. The Higgs boson decays into two photons primarily via either a top quark loop or a W boson loop. The amplitudes of these modes interfere destructively resulting in a branching fraction of around $0.2 \%$ for a SM Higgs boson at $125 \mathrm{GeV}$. Despite the small branching fraction and the presence of a large diphoton continuum background, the diphoton decay mode provides an expected signal significance for the $125 \mathrm{GeV}$ SM Higgs boson that is very high among all the decay modes.

\section{Analysis Strategy}

Photon Identification and Energy Resolution : The photon energy is computed from the sum of the crystal energies recorded by the electromagnetic calorimeter, abbreviated as ECAL, (in the region covered by the preshower detector, $1.65<|\eta|<2.6$, the energy recorded in that detector is added). A multivariate regression technique based on a Boosted Decision Tree (BDT) is deployed to correct the photon energy due to the containment of the shower in the clustered crystals and the shower losses for photons which convert in the material upstream of the calorimeter. In order to obtain the best per-photon energy resolution, the crystal signals are calibrated to compensate several detector effects such as the variation of crystal transparency, the different single-channels response, etc. A photon identification BDT is trained to distinguish prompt photons from fake photons. Fig. 1 shows the BDT output of the photon ID.

Di-photon Vertex Identification : For the better diphoton invariant mass resolution it is very important to find the primary vertex precisely. In order to select the primary vertex from those of other interactions in the same bunch crossing a multivariate analysis (MVA) technique is implemented. If the chosen vertex is within $1 \mathrm{~cm}$ from the true vertex $\left(\left|z_{\text {chosen }}-z_{\text {true }}\right|<1 \mathrm{~cm}\right)$ that is called right vertex (RV). An additional BDT is trained to compute the probability that the chosen vertex was correct (RV). 
Event Classification : BDT technique is also used to identify signal like diphoton pairs. Fig. 2 shows the distribution of the diphoton MVA output for simulated events (stacked histograms) and data (black dots). The variable is built to have a flat distribution on signal events (red shaded histograms). To improve the sensitivity of the analysis, events with additional objects characteristic of different Higgs boson production mechanisms, according to their mass resolution and predicted signal-to-background ratio are classified into 14 mutually exclusive categories. Two categories for events produced in association with $t \bar{t}$ pair depending on the semi-leptonic or hadronic decays of the top quark. Those events in which Higgs bosons are produced in alliance with one of the vector bosons $\left(\mathrm{Z}\right.$ or $\left.W^{ \pm}\right)$are divided into five categories. Three categories are optimized to maximize the expected significance in the VBF production channel. The events which could not be tagged according to the above categories are divided into four untagged categories according to the diphoton mass resolution and predicted signal over background ratio. In Fig. 2 the vertical dashed lines show the boundaries for the definition of the four Untagged categories.

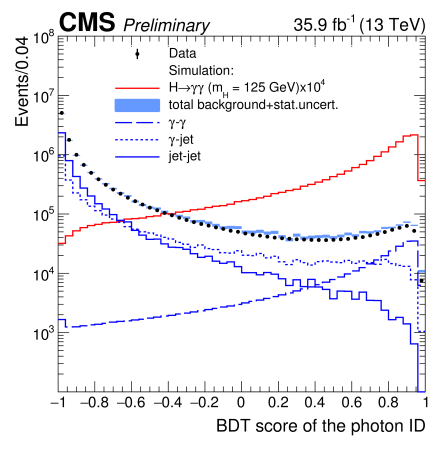

Figure 1: Photon ID BDT score [1]

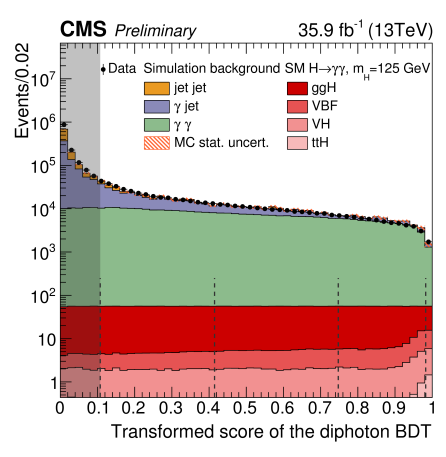

Figure 2: Diphoton MVA output [1]

Signal and Background Modelling : For each process, category and vertex scenario i.e. either right vertex (RV) or wrong vertex, the signal shape for the diphoton invariant mass $\left(m_{\gamma \gamma}\right)$ distribution is constructed from simulation. The $m_{\gamma \gamma}$ distributions are fitted using sum of at most five Gaussian functions. The signal samples at $m_{H}$ in the range 120 to $130 \mathrm{GeV}$ are fitted simultaneously to obtain the parametric variations of the parameters of Gaussian function. Discrete profiling method, as described in [1], is used for background modelling which is data driven. The procedure treats the choice of the background function as a discrete nuisance parameter in the likelihood fit to the data. The list of functions used contains exponentials, Bernstein polynomials, Laurent series and power law functions.

Systematic uncertainties : The following systematic uncertainties have been evaluated. The scale and resolution for the photon's energy, photon identification (preselection and BDT), vertex finding efficiency, trigger efficiency, theoretical production crosssections and uncertainty due to the additional objects in the exclusive categories (jet, leptons and missing energy). The systematic uncertainties on signal strengths and couplings are overtopped typically by the photon shower shape modeling (which affects the photon identification and per photon energy resolution estimate). 


\section{Results}

Expected Event Yields : Fig. 3 shows the expected number of signal events for each category. The total number is broken down by percentage contribution of each production mode to any particular event category. Production processes are separated into the Stage 0 Higgs Simplified Template Cross Section Framework (STXS) [2].

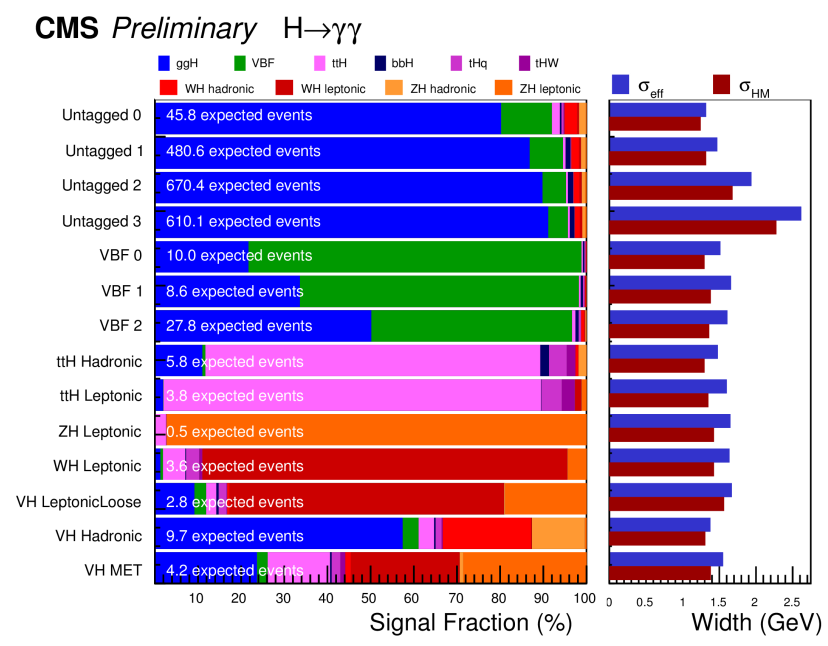

$35.9 \mathrm{fb}^{-1}(13 \mathrm{TeV})$

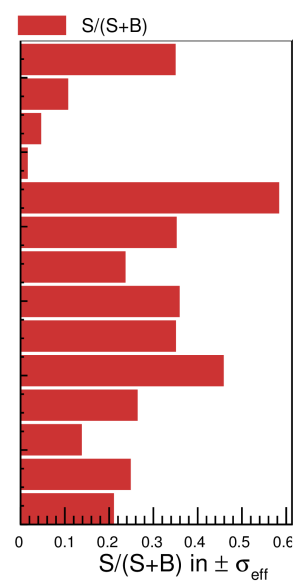

Figure 3: Expected fraction of signal events per production mode in the different categories. For each category, the $\sigma_{e f f}$ and $\sigma_{H M}$ of the signal model are given, as described in the text. The ratio of the number of signal events $(\mathrm{S})$ to the number of signal plus background events $(\mathrm{S}+\mathrm{B})$ is shown on the right hand side. [1]

Signal strength : In Fig. 4 the best fit signal strength modifier for each Higgs boson production process and the best fit signal stregth for all categories combined are shown. Best fit signal strength (XS*BR) relative to $\mathrm{SM}$ is found to be $\hat{\mu}=1.16_{-0.14}^{+0.15}=1.16_{-0.10}^{+0.11}(\text { stat } .)_{-0.08}^{+0.09}(\text { syst } .)_{-0.05}^{+0.06}($ theo. . . The result of a two-dimensional likelihood scan of the signal strength $\mu_{g g h, t \bar{t} H}$ for fermionic production modes (ggH and $t \bar{t} H$ ) and $\mu_{V B F, V H}$ for vector boson production modes (VBF, $\mathrm{ZH}, \mathrm{WH}$ ), with the value of the parameter $m_{H}$, the Higgs boson mass, profiled in the fit, is shown in Fig. 5. The solid and dashed black lines in Fig. 5 demonstrate $1 \sigma$ and $2 \sigma$ contours, respectively. The best-fit values for each modifier are $\mu_{g g H, t \bar{t} H}=1.19_{-0.18}^{+0.20}$ and $\mu_{V B F, V H}=1.01_{-0.51}^{+0.57}$.

Coupling strength modifiers : Two-dimensional likelihood scans of the Higgs boson coupling strength modifiers are produced. $\kappa_{f}$ versus $\kappa_{V}$, the coupling modifiers to bosons and fermions, $\kappa_{\gamma}$ versus $\kappa_{g}$, the effective coupling modifiers to photons and gluons are shown in Fig. 6. The $\kappa$ parameters other than those varied are fixed to 1 in each case. Fig. 6 shows the $1 \sigma$ and $2 \sigma$ contours for each scan and shows the test statistic q, equal to twice the negative log likelihood ratio. The point $\left(\kappa_{V}, \kappa_{f}\right)=(1,-1)$ has an observed (expected) q of 35.6 (43.6), inconsistent with the best fit to an observed (expected) level of $5.6 \sigma(6.3 \sigma)$. 


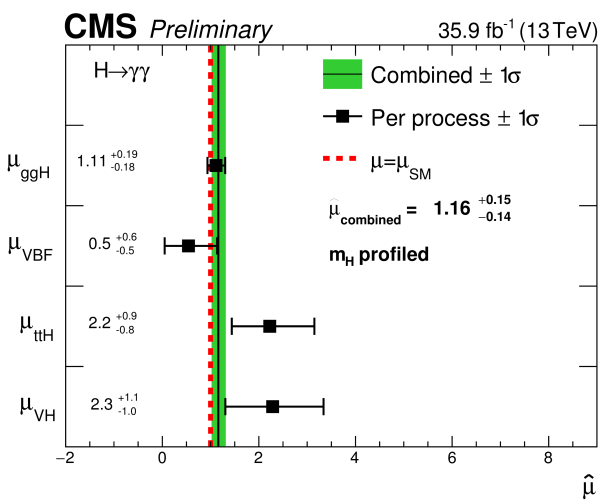

Figure 4: Signal strength modifiers measured for each process (black points for profiled $m_{H}$, compared to the overall signal strength (green band) and to the SM expectation (dashed red line). [1]

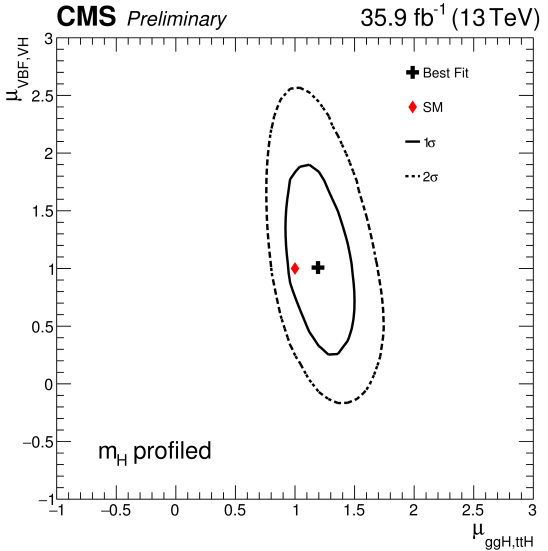

Figure 5: The two-dimensional best-fit (black cross) of the signal strengths for fermionic $(g g H, t \bar{t} H)$ and bosonic $(\mathrm{VBF}$, $\mathrm{ZH}, \mathrm{WH}$ ) production modes compared to the SM expectations (red diamond). The solid (dashed) line represents the 1(2) standard deviation confidence region. [1]

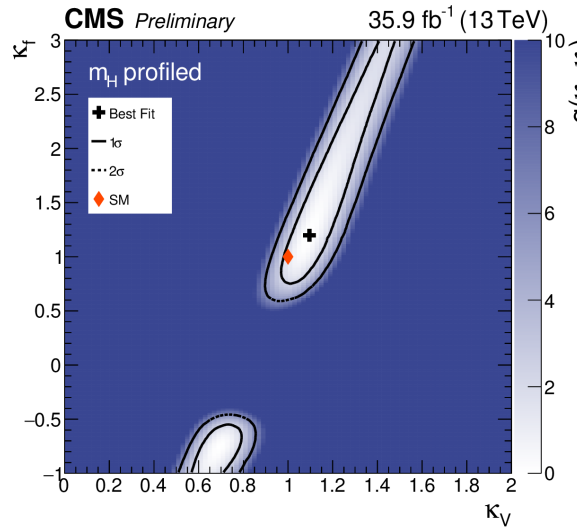

CMS Preliminary

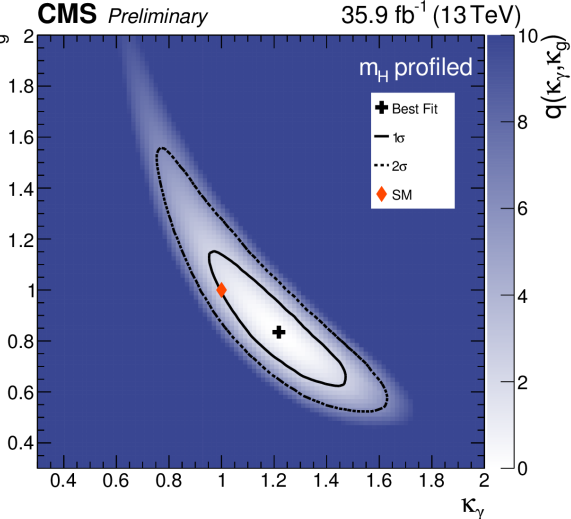

Figure 6: Two-dimensional likelihood scans of $\kappa_{f}$ versus $\kappa_{V}$ (left) and $\kappa_{g}$ versus $\kappa_{\gamma}$ (right). All four variables are expressed relative to the SM expectations. The color maps indicate the value of the test statistic.[1]

\section{References}

[1] CMS Collaboration, "Measurements of properties of the Higgs boson in the diphoton decay channel with the full 2016 data set", CMS Physics Analysis Summary CMS-PAS-HIG-16-040, 2017.

[2] LHC Higgs Cross Section Working Group Collaboration, "Handbook of LHC Higgs Cross Sections: 4. Deciphering the Nature of the Higgs Sector", technical report, 2016. arXiv:1610.07922. 